J. Indones. Math. Soc.

Vol. 18, No. 2 (2012), pp. 67-71.

\title{
CHARACTERISATION OF PRIMITIVE IDEALS OF TOEPLITZ ALGEBRAS OF QUOTIENTS
}

\author{
RIZKY ROSJANUARDI \\ Department of Mathematics Education, \\ Universitas Pendidikan Indonesia (UPI) \\ Jl. Dr. Setiabudhi No. 229 Bandung 40154 \\ rizky@upi.edu
}

\begin{abstract}
Let $\Gamma$ be a totally ordered abelian group, the topology on primitive ideal space of Toeplitz algebras Prim $\mathcal{T}(\Gamma)$ can be identified through the upwards-looking topology if and only if the chain of order ideals is well-ordered. Let $I$ be an order ideal of such that the chain of order ideals of $\Gamma / I$ is not well-ordered, we show that for any order ideal $J \nsupseteq I$, the topology on primitive ideal space can be identified through the upwards-looking topology. Also we discuss the closed sets in Prim $\mathcal{T}(\Gamma)$ with the upwards-looking topology and characterize maximal primitive ideals.
\end{abstract}

Key words: Toeplitz algebra, totally ordered group, primitive ideal, quotient, characterisation.

\begin{abstract}
Abstrak. Misalkan $\Gamma$ adalah grup abel terurut total, topologi pada ruang ideal primitif dari aljabar Toeplitz $\operatorname{Prim} \mathcal{T}(\Gamma)$ dapat diidentifikasi melalui topologi upwards-looking jika dan hanya jika rantai dari ideal urutan adalah terurut dengan rapi (well-ordered). Misalkan $I$ adalah sebuah ideal urutan sedemikian sehingga rantai dari ideal urutan dari $\Gamma / I$ tidak terurut dengan rapi, diperlihatkan bahwa untuk sembarang ideal $J \nsupseteq I$, topologi pada ruang ideal primitif dapat diidentifikasi melalui topologi upwards-looking. Pada paper ini juga dibahas himpunan-himpunan tutup di $\operatorname{Prim} \mathcal{T}(\Gamma)$ di bawah topologi upwards-looking, dan karakterisasi dari ideal primitif maksimal.
\end{abstract}

Kata kunci: Aljabar Toeplitz, grup terurut total, ideal primitif, kuosien, karakterisasi

2000 Mathematics Subject Classification: 46L55.

Received: 28-10-2011, revised: 30-05-2012, accepted: 30-05-2012. 


\section{Introduction}

Suppose $\Gamma$ is a totally ordered abelian group. Let $\Sigma(\Gamma)$ be the chain of order ideals of $\Gamma$, and $X(\Gamma)$ denotes the disjoint union

$$
\bigsqcup\{\hat{I}: I \in \Sigma(\Gamma)\}=\{(I, \gamma): I \in \Sigma(\Gamma), \gamma \in \hat{I}\} .
$$

Adji and Raeburn shows that every primitive ideal of Toeplitz algebra $\mathcal{T}(\Gamma)$ of $\Gamma$ is of the form

$$
\operatorname{ker} Q_{I} \circ \alpha_{\nu}^{\Gamma^{-1}}
$$

where $I$ is an order ideal of $\Gamma$ and $\nu \in \hat{\Gamma}$. They also showed [?, Theorem 3.1] that there is a bijection $L$ of $X(\Gamma)$ onto the primitive ideal space Prim $\mathcal{T}(\Gamma)$ of Toeplitz algebra $\mathcal{T}(\Gamma)$ given by

$$
L(I, \gamma):=\operatorname{ker} Q_{I} \circ \alpha_{\nu}^{\Gamma^{-1}} \text { where } \nu \in \hat{\Gamma} \text { satisfies }\left.\nu\right|_{I}=\gamma .
$$

Adji and Raeburn [?] introduced a topology in $X(\Gamma)$ which is called the upwards-looking topology. When $\Sigma(\Gamma)$ is isomorphic with a subset of $\mathbb{N} \cup\{\infty\}$, the bijection $L$ is a homeomorphism [?, Proposition 4.7], so the usual hull-kernel topology of Prim $\mathcal{T}(\Gamma)$ can be identified through the upwards-looking topology in $X(\Gamma)$. Later, Raeburn and his collaborators [?] showed that $L$ is a homeomorpism if and only if $\Sigma(\Gamma)$ is well-ordered, in the sense that every nonempty subset has a least element.

More recently, Rosjanuardi and Itoh [?] characterised maximal primitive ideals of $\mathcal{T}(\Gamma)$. A series of analysis on subsets of $\Sigma(\Gamma)$ implies that any singleton set $\{\gamma\}$ which consists of a character in $\hat{\Gamma}$ is closed. This implies that every maximal primitive ideal of $\mathcal{T}(\Gamma)$ is of the following form

$$
L(\Gamma, \gamma)=\operatorname{ker} Q_{\Gamma} \circ \alpha_{\gamma}^{\Gamma^{-1}} .
$$

Given a totally ordered abelian group $\Gamma$ and an order ideal $I$. In this paper, we apply the method in [?] and [?] to characterise maximal primitive ideal of Toeplitz algebra $\mathcal{T}(\Gamma / J)$ of quotient $\Gamma / J$ when the chain of order ideal $\Sigma(\Gamma / I)$ is not well-ordered.

\section{UPWARDS-LOOKING TOPOLOGY}

Let $\Gamma$ be a totally ordered abelian group. The Toeplitz algebra $\mathcal{T}(\Gamma)$ of $\Gamma$ is the $\mathrm{C}^{*}$-subalgebra of $B\left(\ell^{2}\left(\Gamma^{+}\right)\right)$generated by the isometries $\left\{T_{x}=T_{x}^{\Gamma}: x \in \Gamma^{+}\right\}$ which are defined in terms of the usual basis by $T_{x}\left(e_{y}\right)=e_{y+x}$. This algebra is universal for isometric representation of $\Gamma^{+}[?$, Theorem 2.9].

Let $I$ be an order ideal of $\Gamma$. Then the map $x \longmapsto T_{x+I}^{\Gamma / I}$ is an isometric representation of $\Gamma^{+}$in $\mathbb{T}(\Gamma / I)$. Therefore by the universality of $\mathcal{T}(\Gamma)$, there is a homomorphism $Q_{I}: \mathcal{T}(\Gamma) \longrightarrow \mathcal{T}(\Gamma / I)$ such that $Q_{I}\left(T_{x}\right)=T_{x+I}^{\Gamma / I}$, and that $Q_{I}$ is surjective. Suppose $\mathcal{C}(\Gamma, I)$ denotes the ideal in $\mathcal{T}(\Gamma)$ generated by $\left\{T_{u} T_{u}^{*}-T_{v} T_{v}^{*}\right.$ : 
$\left.v-u \in I^{+}\right\}$and $\operatorname{Ind}_{I^{\perp}}^{\hat{\Gamma}}\left(\mathcal{T}(\Gamma / I), \alpha^{\Gamma / I}\right)$ is the closed subalgebra of $C(\hat{\Gamma}, \mathcal{T}(\Gamma / I))$ satisfying $f(x h)=\alpha_{h}^{\Gamma / I^{-1}}(f(x))$ for $x \in \hat{\Gamma}, h \in I^{\perp}$. It was proved in [?, Theorem 3.1] that there is a short exact sequence of $C^{*}$-algebras:

$$
0 \rightarrow \mathcal{C}(\Gamma, I) \rightarrow \mathcal{T}(\Gamma) \stackrel{\phi_{I}}{\rightarrow} \operatorname{Ind}_{I^{\perp}}^{\hat{\Gamma}}\left(\mathcal{T}(\Gamma / I), \alpha^{\Gamma / I}\right) \rightarrow 0
$$

in which $\phi_{I}(a)(\gamma)=Q_{I} \circ\left(\alpha_{\gamma}^{\Gamma}\right)^{-1}(a)$ for $a \in \mathcal{T}(\Gamma), \gamma \in \hat{\Gamma}$, and $\alpha_{\gamma}$ is dual action of $\hat{\Gamma}$ on $\mathcal{T}(\Gamma)$ characterized by $\alpha_{\gamma}^{\Gamma}\left(T_{x}\right)=\gamma(x) T_{x}$. The identity representation $T^{\Gamma / I}$ of $\mathcal{T}(\Gamma / I)$ is irreducible [?], it follows from [?, Proposition 6.16] that $\operatorname{ker} Q_{I} \circ\left(\alpha_{\gamma}^{\Gamma}\right)^{-1}$ is a primitive ideal of $\mathcal{T}(\Gamma)$.

If $X(\Gamma)$ denotes the disjoint union

$$
\bigsqcup\{\hat{I}: I \in \Sigma(\Gamma)\}=\{(I, \gamma): I \in \Sigma(\Gamma), \gamma \in \hat{I}\},
$$

it was showed in [?, Theorem 3.1] that

$$
L(I, \gamma):=\operatorname{ker} Q_{I} \circ \alpha_{\nu}^{\Gamma^{-1}} \text { where } \nu \in \hat{\Gamma} \text { satisfies }\left.\nu\right|_{I}=\gamma,
$$

is a bijection of $X(\Gamma)$ onto $\operatorname{Prim} \mathcal{T}(\Gamma)$.

Using the bijection $L$, Adji and Raeburn describe a new topology on $X$ which corresponds to the hull-kernel topology on Prim $\mathcal{T}(\Gamma)$. This new topology, is later called the upwards-looking topology. They topologise $X$ by specifying the closure operation as stated in the following definition.

Definition 2.1. [?] The closure $\bar{F}$ of a subset $F$ of $X$ is the set consisting of all pairs $(J, \gamma)$ where $J$ is an order ideal and $\gamma \in \hat{J}$ such that for every open neighbourhood $N$ of $\gamma$ in $\hat{J}$, there exists $I \in \Sigma(\Gamma)$ and $\chi \in N$ for which $I \subset J$ and $\left(I,\left.\chi\right|_{I}\right) \in F$.

Example 2.2. [?, Example 3] We are going to discuss some description of sets in $X(\Gamma)$ by considering specific cases of $\Gamma$. An observation on $\Gamma:=\mathbb{Z} \oplus_{\text {lex }} \mathbb{Z}$ gives interesting results. Let $I$ be the ideal $\{(0, n): n \in \mathbb{Z}\}$, since $I$ is the only ideal, we have $X(\Gamma)=\hat{0} \sqcup \hat{I} \sqcup \hat{\Gamma}$. Suppose $\lambda_{0}$ is a character in $\hat{I}$ defined by $(0, n) \mapsto e^{2 \pi i n}$, and let $F=\left\{\lambda_{0}\right\}$. Next we consider a character $\gamma$ in $\hat{\Gamma}$ defined by $(m, n) \mapsto e^{2 \pi i(m+n)}$. It is clear that $\left.\gamma\right|_{I}=\lambda_{0}$. Then $\gamma \in \bar{F}$, because every open neighbourhood $N$ of $\gamma$ in $\hat{\Gamma}$ contains an element $\lambda$ (which is nothing but $\gamma$ it self) such that its restriction on I gives a character in $F$. It is clear that $\gamma \notin F$, hence $F$ is not closed in the upwards-looking topology for $X(\Gamma)$.

Adji and Raeburn [?] proved that this is the correct topology to identify the hull-kernel topology of Prim $\mathcal{T}(\Gamma)$ when $\Gamma$ is a group such that the set $\Sigma(\Gamma)$ of order ideal is order isomorphic to a subset of $\mathbb{N} \cup\{\infty\}$. In [?], Raeburn and his collaborators extended the results in [?]. Their main theorem, says that $\operatorname{Prim} \mathcal{T}(\Gamma)$ is homeomorphic to $X(\Gamma)$ with the upwards-looking topology if and only if the totally ordered set $\Sigma(\Gamma)$ is well-ordered in the sense that every non-empty subset has a least element. Their technique uses classical Toeplitz operators as well as the 
universal property of $\mathcal{T}(\Gamma)$ which was the main tool in [?]. Then they described $\operatorname{Prim} \mathcal{T}(\Gamma)$ when parts of $\Sigma(\Gamma)$ are well-ordered.

Rosjanuardi in [?] improved the results in [?] to the case when $\Sigma(\Gamma)$ is not well ordered. In [?, Proposition 6] it is stated that when $\Sigma(\Gamma)$ is isomorphic to a subset of $\{-\infty\} \cup \mathbb{Z} \cup\{\infty\}$, then we can use the upwards-looking topology on $X(\Gamma / I)$ to identify the topology on $\operatorname{Prim} \mathcal{T}(\Gamma / I)$. For general totally abelian group $\Gamma$, as long as there is an order ideal $I$ such that every order ideal $J \supseteq I$ has a successor, the upwards-looking topology is the correct topology for Prim $\mathcal{T}(\Gamma / I)$ [?, Proposition 8]. In [?, Theorem 9] it was proved that for any quotient $\Gamma / I$ such that the chain $\Sigma(\Gamma / I)$ is isomorphic to a subset $\{-\infty\} \cup \mathbb{Z} \cup\{\infty\}$, for any order ideal $J \supsetneqq I$, the upwards-looking topology on $\Sigma(\Gamma / J)$ is the correct topology for Prim $\mathcal{T}(\Gamma / J)$.

\section{Characterisation of Primitive Ideals}

Example ?? implies that any closed set in the point wise topology is not necessarily closed in the upwards-looking topology. When it is applied to any complement $F^{C}$ of a set $F$, it arrives to a conclusion that any open set in the point wise topology, is not necessarily open in the upwards-looking topology. This example motivated Rosjanuardi and Itoh [?] to prove more general cases.

Combining results in [?] with ones in [?] give characterisation results for more general cases than in [?].

Proposition 3.1. Suppose that $\Gamma$ is a totally ordered abelian group such that the chain $\Sigma(\Gamma)$ of order ideals in $\Gamma$ is isomorphic to a subset of $\{-\infty\} \cup \mathbb{Z} \cup\{\infty\}$. For any $I \in \Sigma(\Gamma)$, the maximal primitive ideals of $\mathcal{T}(\Gamma / I)$ are of the form

$$
\operatorname{ker} Q_{\Gamma / I} \circ\left(\alpha_{\gamma}^{\Gamma / I}\right)^{-1} \text {. }
$$

Proof. Let $I \in \Sigma(\Gamma)$. The chain of order ideals in $\Gamma / I$ is

$$
I \subset J_{1} / I \subset J_{2} / I \subset \ldots,
$$

where $J_{i} \in \Sigma(\Gamma)$ and $I \subset J_{i} \subset J_{i+1}$ for all $i$. Hence $\Sigma(\Gamma / I)$ is well ordered. Give the set $X(\Gamma / I):=\bigsqcup\{\widehat{J / I}: J \in \Sigma(\Gamma), I \subset J\}$ the upwards-looking topology, hence $L^{\Gamma / I}$ is a homeomorphism of $X(\Gamma / I)$ onto $\operatorname{Prim} \mathcal{T}(\Gamma / I)$ by Theorem 3.1 of [?]. Proposition 6 of [?], then implies that $X(\Gamma / I)$ is homeomorphic with $\operatorname{Prim}(\mathcal{T}(\Gamma))$. Theorem 11 of [?] then gives the result.

Proposition 3.2. Suppose that $\Gamma$ is a totally ordered abelian group, and let $I$ be an order ideal in $\Gamma$ such that every oreder ideal $J \supseteq I$ has a successor. Then the maximal primitive ideals of $\mathcal{T}(\Gamma / I)$ are of the form

$$
\operatorname{ker} Q_{\Gamma / I} \circ\left(\alpha_{\gamma}^{\Gamma / I}\right)^{-1} \text {. }
$$

Proof. Let $I \in \Sigma(\Gamma)$ such that every order ideal $J \supseteq I$ has a successor. Since each nontrivial element of $\Sigma(\Gamma / I)$ is of the form $J / I$ for $J \in \Sigma(\Gamma)$ and $J \supsetneq I$, every element of $\Sigma(\Gamma / I)$ has a successor. This implies that $\Sigma(\Gamma / I)$ is well ordered. Give the set $X(\Gamma / I):=\bigsqcup\{\widehat{J / I}: J \in \Sigma(\Gamma), I \subset J\}$ the upwards-looking topology, hence 
$L^{\Gamma / I}$ is a homeomorphism by Theorem 3.1 of [?]. The result then follows from Theorem 9 of [?].

Theorem 3.3. Suppose that $\Gamma$ is a totally ordered abelian group, and $I \in \Sigma(\Gamma)$ such that $\Sigma(\Gamma / I) \cong\{-\infty\} \cup \mathbb{Z} \cup\{\infty\}$. Let $J \in \Sigma(\Gamma)$ such that $J \supsetneqq I$. Then the maximal primitive ideals of $\mathcal{T}(\Gamma / J)$ are of the form

$$
\operatorname{ker} Q_{\Gamma / J} \circ\left(\alpha_{\gamma}^{\Gamma / J}\right)^{-1} \text {. }
$$

Proof. Since every nontrivial ideal of $\Gamma / I$ is of the form $J / I$ where $J \in \Sigma(\Gamma)$ and $J \nsupseteq I$ and for ideals $J_{1}, J_{2}$ such that $J_{1} / I \subseteq J_{2} / I$ implies $J_{1} \subseteq J_{2}$, then may write

$$
\Sigma(\Gamma / I):=\left\{I=J_{-\infty} \subseteq \ldots \subseteq J_{k} / I \subseteq J_{k+1} / I \subseteq \ldots \subseteq \Gamma=J_{\infty}\right\} .
$$

Now consider the subset

$$
\mathcal{I}:=\left\{I=J_{-\infty} \subseteq \ldots \subseteq J_{k} \subseteq J_{k+1} \subseteq \ldots \subseteq J_{\infty}=\Gamma\right\}
$$

of $\Sigma(\Gamma)$. If $J \neq I$ is an element of $\mathcal{I}$, i.e $J \in \Sigma(\Gamma)$ such that $J \supsetneqq I$, the set

$$
\Sigma(\Gamma / J)=\left\{J \subseteq K_{1} / J \subseteq K_{2} / J \ldots\right\}
$$

is well ordered. Hence $L^{\Gamma / J}$ is a homeomorphism of $X(\Gamma / J)$ onto Prim $\mathcal{T}(\Gamma / J)$ by Theorem 3.1 of [?]. The result is then follow from Theorem 9 of [?].

Acknowledgement. This paper was completed when the author visited the Department of Mathematics, Gunma University, Maebashi-Japan, 2011. Supported by Gunma University and UPI research fund. The author would thank to these institutions.

\section{References}

[1] S. Adji, Semigroup crossed products and the structure of Toeplitz algebras, J. Op. Theory 44 (2000), 139-150.

[2] S. Adji and I. Raeburn, The ideal structure of Toeplitz algebra, Integral Equation Operation Theory 48 (2004), 281-293.

[3] S. Adji, I. Raeburn and R. Rosjanuardi, Group extensions and the primitive ideal spaces of Toeplitz algebras, Glasgow Math. Journal 49 (2007), 81-92.

[4] G.J. Murphy, Ordered group and Toeplitz algebras, J. Operator Theory 18 (1987), 303-326.

[5] R. Rosjanuardi, Primitive ideals of Toeplitz algebra of ordered groups, J. Indones. Math. Soc. (MIHMI) Vol. 14 No. 2 (2008), 111-118.

[6] R. Rosjanuardi and T. Itoh, Characterisation of maximal primitive ideals of Toeplitz algebras, Scientiae Mathematicae Japonicae, Vol. 72, No. 2. (2010), 121-126.

[7] D.P. William and I. Raeburn, Morita equivalence and continuous-trace $C^{*}$-algebras, Amer. Math. Soc., Providence, RI (1998). 